\title{
Process Modelling - What Really Matters
}

\author{
Michael Rosemann \\ Faculty of Science and Technology, Queensland University of Technology \\ 126 Margaret Street, Brisbane Qld 4000, Australia \\ m.rosemann@qut. edu. au
}

\begin{abstract}
Process modelling has become one of the most popular forms of conceptual modelling. However, there is an increasing body of evidence suggesting that the requirements of organisations and the focus of related academic research do not sufficiently overlap. This keynote presentation will start with the results of a comparative study on the benefits, issues and challenges as they are perceived by academics, IT vendors and end users. It will become obvious that there is a substantial gap between the priorities of these communities. Recommendations and specific examples will be provided for how to close this gap in order to increase the relevance of research on process modelling. This will cover context-aware process modelling, collaborative process modelling, visualisation and the overall success of process modelling. As a consequence, it will be postulated to be more proactive in terms of collaborations between designoriented and behavioural researchers.
\end{abstract}

\section{Bio}

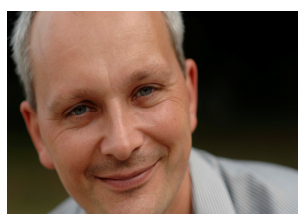

Dr Michael Rosemann is a Professor for Information Systems and Co-Leader of the Business Process Management (BPM) Group at Queensland University of Technology, Brisbane, Australia. He is the Chief Investigator of a number of applied research projects funded by the Australian Research Council (ARC) and various industry partners. He was a member of the ARC College of Experts in 2006/07.

A prolific writer, he is the author/editor of seven books, more than 140 refereed papers (incl. MISQ, JAIS, IEEE TKDE, Information Systems) and Editorial Board member of seven international journals. His publications have been translated into German, Russian, Portuguese and Chinese. Michael's PhD students have won the Australian award for the best PhD thesis in Information Systems in 2007 and in 2008. Michael is the co-inventor of seven US patent proposals related to process modelling. Dr Rosemann is the founder and chair of the Australian BPM Community of Practice (bpm-collaboration.com) and he has been the Chair of the $5^{\text {th }}$ International Business Process Management Conference in 2007. He regularly conducts executive training in BPM (www.bpm-training.com) and provided advice to organisations from various industries including telecommunications, banking, insurance, utility, retail, public sector, logistics and film industry. 